\title{
Feedback Control for Distributed Heat Transfer Mechanisms in Direct-Contact Membrane Distillation System
}

\author{
Fadi Eleiwi, Ibrahima N'Doye and Taous-Meriem Laleg-Kirati \\ \{fadi.eleiwi, ibrahima.ndoye, taousmeriem.laleg@kaust.edu.sa\}
}

\begin{abstract}
In this paper, the problem of stabilization and production rate reference tracking for a Direct-Contact Membrane Distillation (DCMD) system is addressed. Sufficient conditions for the asymptotic and exponential stabilization for DCMD system are presented using the Gronwall-Bellman lemma and Linear Matrix Inequalities (LMIs) approaches, respectively. A nonlinear observer is then proposed to estimate the temperature distribution among the DCMD domain. This contributes to propose a reference production rate control design for the DCMD process via observer-based output control approach. Finally, numerical simulations are given to show the effectiveness of the proposed methods.
\end{abstract}

Index Terms-Direct-Contact Membrane Distillation (DCMD), feedback stabilization, reference output tracking, static state feedback, observers design, observer based output feedback, LMIs.

\section{INTRODUCTION}

Membrane distillation (MD) is a promising water desalination method with excellent potential for producing clean water in multiple applications [1]. MD is based on thermal separation, utilizing micro-porous membrane as an in-between medium which lets only water vapor and other volatile molecules transfer through the membrane's dry pores. The transfer happens from a feed container of high temperature solution to a permeate container with a relatively lower temperature to produce clean water (see [1]-[4] and references therein). The process is driven by a pressure gradient across the membrane, whereas the gradient is driven by the difference in temperature along the membrane boundary layers [5]. This sheds light on the significance of the temperature distribution in the MD module, especially along the surfaces of the membrane [6]. MD has four common configurations, in which they share the same principle of operation in the feed side. They differ, however, in the methodology of water vapor condensation in the permeate side. The most popular configuration for MD is DirectContact Membrane Distillation (DCMD), where both the feed and permeate solutions are in direct contact with the membrane sides. This adds simplicity in an experimental laboratory setup [1], [5]. The DCMD configuration is the subject of study and analysis in this paper.

Despite of the attractive nature of the DCMD process and unlike other water desalination methods, such as reverse osmosis (RO) [7], few works have been proposed to control

Fadi Eleiwi, Ibrahima N'Doye and Taous-Meriem Laleg-Kirati are with Computer, Electrical and Mathematical Sciences and Engineering Division (CEMSE), King Abdullah University of Science and Technology(KAUST). the production rate of the DCMD [6]. Controlling the DCMD production rate, along with having an acceptable energy consumption rate, has the potential to lead to commercialization in industrial applications. Achieving a desired production rate of clean water is acquirable by controlling the temperatures difference of the membrane boundary layers, due to their significance in driving the process [8]. In this paper, we propose the use of feedback stabilization techniques to track a desired production rate reference through the temperature difference of the membrane boundary layers. We use a nonlinear 2D-Advection-Diffusion Equation dynamic model (ADE) for the DCMD process that is presented in [4], [9].

Several studies on the control and state estimation of nonlinear system exist in the literature. An example of the proposed methods, the linearization approach or Lyapunov theories (see [10]-[19] and references therein).

Another approach to stabilize nonlinear systems is based on the Gronwall-Bellman lemma [20]-[22]. This lemma has been applied to the exponential stability of nonlinear affine systems [23], to the nonlinear observer design [24], to the robust stabilization and observation of nonlinear uncertain systems [25], to the robust stability of linear systems [26], [27] and to finite-time stability of linear singular systems [28]. The exponential stabilization of a class of nonlinear systems through the use of a generalized Gronwall-Bellman lemma approach has been investigated as well [29]. It is well-known that the Gronwall-Bellman inequality provides an explicit bound to an unknown function. It is as well a powerful tool in the study of quantitative properties and the stability of solutions to differential and integral equations. Another technique is the Linear Matrix Inequality (LMI) [30], where it has attracted more attention and has a wide range of applications. This is due to its high success rate in the analysis and design in the control system, which in particular has an advantage in dealing with nonlinear systems [30].

The aim of this paper is to develop sufficient conditions for reference tracking in DCMD process using the GronwallBellman lemma approach and the LMI formulation. The nonlinear system considered is Lipschitz in the state and the proposed stabilization methods are simple to implement in practice.

In this paper, the 2D ADE dynamic model for the DCMD process is described in section II. In section III, sufficient conditions for the asymptotic and exponential stabilization of the DCMD process are derived in terms of LMI formulation 
and Gronwall-Bellman lemma respectively. The GronwallBellman lemma establishes a set of norm criteria of the exponential stability of the DCMD process by considering the static state feedback control. Later, the design of the observer-based output feedback control for reference tracking is described. Numerical simulations are presented in section IV to illustrate the application of these proposed approaches to the nonlinear model of DCMD process.

Notations. $\|x\|=\sqrt{x^{T} x}$ and $\|A\|=\sqrt{\lambda_{\max }\left(A^{T} A\right)}$ are the Euclidean vector norm and the spectral matrix norm respectively, where $\lambda_{\max }\left(A^{T} A\right)$ is the maximal eigenvalue of the symmetric matrix $A^{T} A$.

\section{DCMD MODEL}

The DCMD process is best described with dynamic models which are able to examine the transient and steady-state behavior of the different heat transfer mechanisms inside the DCMD process. In this context, we use the recently developed model of the 2D ADE as the DCMD process model. This model is presented in [6], [9].

\section{A. Mathematical model of the DCMD process}

The DCMD process is modeled with 2D ADE model which contributes toward a comprehensive timeline description for the different heat transfer mechanisms inside the DCMD process. Nevertheless, it provides a rigorous behavior description for the conduction and convection heat transfer mechanisms throughout the transient and the steady-state phases. The DCMD model is in (1).

with

$$
\begin{array}{r}
\frac{\partial T(x, z, t)}{\partial t}+u_{z} \frac{\partial T(x, z, t)}{\partial z}+u_{x} \frac{\partial T(x, z, t)}{\partial x} \\
=\alpha_{x} \frac{\partial^{2} T(x, z, t)}{\partial x^{2}}+\alpha_{z} \frac{\partial^{2} T(x, z, t)}{\partial z^{2}},
\end{array}
$$

$$
0<t<\tau_{\text {final }}, \quad x \in\left[0, x_{f m}\right] \cup\left[x_{p m}, X\right], \quad 0<z<Z .
$$

where $T$ is the temperature distribution among the domain of the DCMD module, $u_{x}$ and $u_{z}$ are the water stream flow rates along the $x, z$ axes, respectively. $\alpha_{x}$ and $\alpha_{z}$ are constants that depend on the thermal conductivity $\kappa_{e}$, specific heat $c_{p}$, and the density $\rho$ of the used water, as shown in (2).

$$
\alpha=\frac{\kappa_{e}}{\rho c_{p}} .
$$

The model solves for the temperature time evolution inside the designated process domain, in which $T(x, z, t)$ is the variable of interest, with the inlet temperatures of the feed and permeate containers as the process inputs. The model is studied in a rectangular domain, shown in Fig. 1, with final values for the time, and the space coordinates.

The initial conditions of the model are

$$
T_{f}(x, z, 0)=T_{f_{\text {initial }}} \text { and } T_{p}(x, z, 0)=T_{p_{\text {initial }}},
$$

where the associated boundary conditions with the dynamic model (e.g. feed solution $f$ ) are set to be a Dirichlet condition for the inlet temperature of each subsystem like in (4), (5), and Neumann conditions for the rest of the boundaries, as in (6), (7), (8), (9).

$$
T(x, 0, t)=T_{0_{f}} \quad \text { for } \quad x \in\left[0, x_{f m}\right]
$$

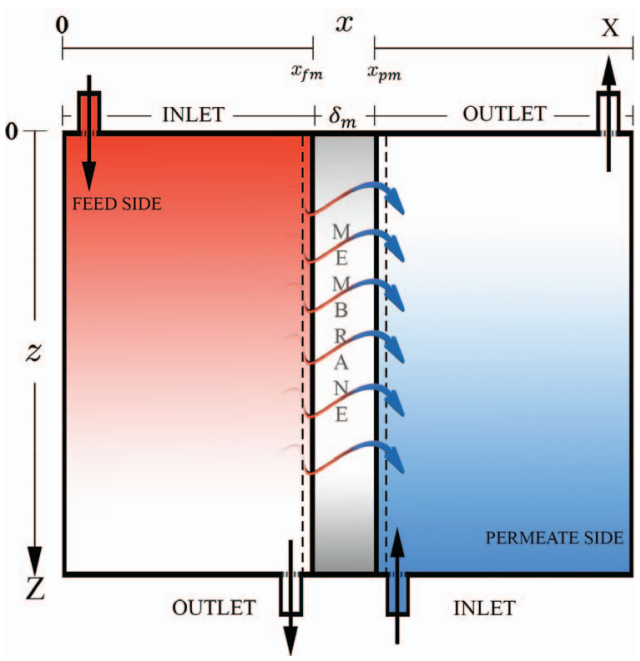

Fig. 1. DCMD counter current schematic diagram with the inlets and outlets of feed/permeate water.

$$
\begin{aligned}
T(x, Z, t) & =T_{0_{p}} \text { for } \quad x \in\left[x_{p m}, X\right] \\
\left.\frac{\partial T(x, z, t)}{\partial x}\right|_{x=0} & =0,\left.\quad \frac{\partial T(x, z, t)}{\partial x}\right|_{x=X}=0 \\
\left.\frac{\partial T(x, z, t)}{\partial x}\right|_{x=x_{f m}} & =\left[J(T) H(T)+\frac{k_{m}}{\delta_{m}} T\left(x_{f m}, z, t\right)\right. \\
& \left.-\frac{k_{m}}{\delta_{m}} T\left(x_{p m}, z, t\right)\right] / k_{f} \\
\left.\frac{\partial T(x, z, t)}{\partial x}\right|_{x=x_{p m}} & =\left[J(T) H(T)+\frac{k_{m}}{\delta_{m}} T\left(x_{f m}, z, t\right)\right. \\
\left.\frac{\partial T(x, z, t)}{\partial z}\right|_{z=Z} & \left.=\frac{k_{m}}{\delta_{m}} T\left(x_{p m}, z, t\right)\right] / k_{p} \\
& \left.\phi \frac{\partial T(x, z, t)}{\partial z}\right|_{z=0}=\phi
\end{aligned}
$$

where $k_{m}$ is the average thermal conductivity of membrane and vapor, $\delta_{m}$ is the membrane thickness, $\phi \in \mathbb{R}^{+}$is a positive constant to represent the amount of flux that is released outside the process containers. $H(T)$ represents the latent heat of evaporation that is responsible of the released sensible heat as in (10).

$$
H(T)=2500.8-2.36 T+0.0016 T^{2}-0.00006 T^{3} .
$$

$J(T)$ is the trans-membrane heat flux transferred between the feed and the permeate subsystems, it is based on Knudsen model for the mass transfer coefficient [1].

$$
J(T)=1.064 \frac{r \epsilon}{\chi \delta_{m}}\left(\frac{M}{R T_{\text {mean }}}\right)^{0.5}\left(P_{f}-P_{p}\right),
$$

where $r$ is the pore size, $\epsilon$ is the porosity, $\chi$ is the tortuosity, $M$ is the water molecular weight, $P$ is the membrane boundary layer pressure, and $R$ is the universal gas constant.

\section{B. Semi-discrete model}

The 2D ADE model in (1) is semi-discretized, using finite difference schemes, and transformed to a high dimension set of ordinary differential equations in (12) as described in [6].

$$
\left\{\begin{array}{l}
\dot{\mathbf{T}}=\mathbb{A} \mathbf{T}+\mathbb{B} U+f(\mathbf{T}), \\
\zeta=\mathbb{H} \mathbf{T},
\end{array}\right.
$$


where vector $\mathbf{T} \in \mathbb{R}^{2 n^{2} \times 1}$ is the state vector that represents the temperature distribution at each grid point inside the feed $T_{f}$ and the permeate $T_{p}$ designated domains.

$$
\mathbf{T}=\left[\begin{array}{c}
T_{f} \\
T_{p}
\end{array}\right]
$$

and thus $T_{f}, T_{p} \in \mathbb{R}^{n^{2} \times 1}$ with $n$ is the number of discretization points in $x$ and $z$ coordinates. Matrix $\mathbb{A} \in \mathbb{R}^{2 n^{2} \times 2 n^{2}}$ represents the operator matrix that has the dynamics of the feed and the permeate subsystems with their mutual couplings on the boundaries (14), where $i, j$ are the discretization grid indices, $A_{f} \in \mathbb{R}^{n^{2} \times n^{2}}, A_{p} \in \mathbb{R}^{n^{2} \times n^{2}}$, $C_{f p} \in \mathbb{R}^{n^{2} \times n^{2}}$, and $C_{p f} \in \mathbb{R}^{n^{2} \times n^{2}} . U(t)$ is the input vector that contains feed and permeate temperature inlets as shown in (15). Matrix $B \in \mathbb{R}^{2 n^{2} \times 2}$ is the input matrix, as shown in (16) (for more details, see [6]).

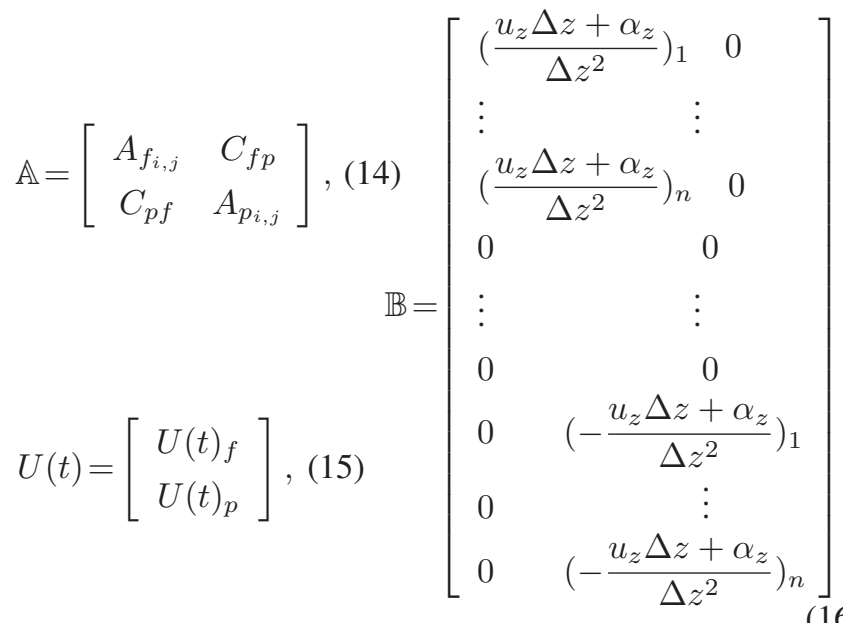

$\zeta$ is the measured output and $\mathbb{H} \in \mathbb{R}^{1 \times 2 n^{2}}$ is the output matrix defined as

$$
\mathbb{H}=[0 \cdots 0110 \cdots 0],
$$

and $f(\mathbf{T}) \in \mathbb{R}^{2 n^{2} \times 1}$ includes the different boundary conditions of each subsystem, notably ones associated with the membrane boundary layers. Some of the boundary conditions are nonlinear and functions of the state vector $\mathbf{T}$, as shown in (8) and (9). However it is obvious that $f(\mathbf{T})$ is globally Lipshitz, where it satisfies the following Lipshitz constraint:

$$
\left\|f\left(\mathbf{T}_{1}\right)-f\left(\mathbf{T}_{2}\right)\right\| \leqslant \lambda\left\|\mathbf{T}_{1}-\mathbf{T}_{2}\right\|,
$$

where $\lambda>0$ is a known Lipschitz constant.

In the following section, we present a feedback stabilization procedure along with observer design that will be used to control the production rate in the DCMD process. This is achieved by maintaining the linear combination of the temperature difference between the membrane boundary layers at a desired and sufficient reference.

\section{FEedback Stabilization And ReFEREnCE OUTPUT TRACKING OF DCMD PROCESS}

First, we present the state feedback control on the nonlinear system described by (12), where it is stated to be globally Lipshitz and the pair $(\mathbb{A}, \mathbb{B})$ stabilizable and the pair $(\mathbb{H}, \mathbb{A})$ is observable.

\section{A. State feedback Stabilization}

The following theorem gives the asymptotic and exponential state feedback stabilization of system (12) using the LMI and Gronwall-Bellman approaches respectively.

\section{Theorem 1:}

i) System (12) controlled using the linear static state feedback

$$
U=-\mathbb{L} \mathbf{T}
$$

is asymptotically stable. If there exist matrices $Q=$ $Q^{T}>0, W$ and a positive scalar $\varepsilon$ such that the following linear matrix inequality (LMI) is satisfied:

$$
\left[\begin{array}{cc}
Q \mathbb{A}^{T}+\mathbb{A} Q-W^{T} \mathbb{B}^{T}-\mathbb{B} W+\varepsilon \lambda^{2} I & Q \\
Q & -\varepsilon I
\end{array}\right]<0 .
$$

The stabilizing state feedback gain matrix is given by

$$
\mathbb{L}=W Q^{-1} .
$$

ii) System (12) controlled using the state feedback (19), is exponentially stable if all eigenvalues of matrix $\mathbb{A}-\mathbb{B L}$ have a strictly negative real part, then there exits two reals, $M>0$ and $\omega<-\lambda M<0$, such that

$$
\left\|e^{(\mathbb{A}-\mathbb{B L}) t}\right\|<M e^{\omega t} \quad \forall t \geqslant 0
$$

and if

$$
0<\left\|\mathbf{T}_{0}\right\| \leqslant \varepsilon_{0}
$$

where $\varepsilon_{0}>0$ is a small scalar.

Moreover, the state $\mathbf{T}(t)$ is bounded by

$$
\|\mathbf{T}(t)\| \leqslant M \varepsilon_{0} \exp (\lambda M+\omega) t .
$$

To prove the theorem the following lemmas are used:

Lemma 1: [31] Let $X$ and $Y$ be real vectors of the same dimension. Then, for any scalar $\varepsilon>0$, the following inequality holds:

$$
X^{T} Y+Y^{T} X \leqslant \varepsilon X^{T} X+\varepsilon^{-1} Y^{T} Y .
$$

Lemma 2 (Gronwall-Bellman lemma): [22] (p 236) [21] (p 252) Let $k: \mathbb{R}^{+} \mapsto \mathbb{R}$, locally integrable on $\mathbb{R}^{+}, k \geqslant 0$, and $c$ be a real constant.

If $u: \mathbb{R}^{+} \mapsto \mathbb{R}$ satisfies

$$
u(t) \leqslant c+\int_{0}^{t} k(\tau) u(\tau) \mathrm{d} \tau, \quad \forall t \geqslant 0
$$

then

$$
u(t) \leqslant c \exp \left(\int_{0}^{t} k(\tau) \mathrm{d} \tau\right), \quad \forall t \geqslant 0 .
$$

Proof: Using the linear state feedback control law (19), the nonlinear system can be written as

$$
\dot{\mathbf{T}}=(\mathbb{A}-\mathbb{B L}) \mathbf{T}+f(\mathbf{T}) .
$$

Consider the following Lyapunov function candidate:

$$
V=\mathbf{T}^{T} P \mathbf{T} \text {. }
$$

Taking the time derivative of $V$ along the solution trajectories of (27) and substituting (27) into (28), we obtain

$$
\begin{aligned}
\dot{V} \leqslant \mathbf{T}^{T}\left((\mathbb{A}-\mathbb{B L})^{T} P+P(\mathbb{A}-\mathbb{B} \mathbb{L})\right) \mathbf{T} \\
+\mathbf{T}^{T} P f(\mathbf{T})+f(\mathbf{T})^{T} P \mathbf{T} .
\end{aligned}
$$


By using relation (24), we obtain the following inequality: $\mathbf{T}^{T} P f(\mathbf{T})+f(\mathbf{T})^{T} P \mathbf{T} \leqslant \varepsilon f(\mathbf{T})^{T} f(\mathbf{T})+\varepsilon^{-1} \mathbf{T}^{T} P P \mathbf{T}$.

By taking into account the Lipschitz condition (18), equation (29) can be written as

$$
\dot{V} \leqslant \mathbf{T}^{T}\left((\mathbb{A}-\mathbb{B L})^{T} P+P(\mathbb{A}-\mathbb{B L})+\varepsilon^{-1} P P+\varepsilon \lambda^{2} I\right) \mathbf{T} .
$$

If

$$
(\mathbb{A}-\mathbb{B} \mathbb{L})^{T} P+P(\mathbb{A}-\mathbb{B L})+\varepsilon^{-1} P P+\varepsilon \lambda^{2} I<0,
$$

then $\dot{V}<0$, which implies that the closed-loop system (27) is asymptotically stable.

Taking into account the inequality in (32), we define $Q=$ $P^{-1}, \mathbb{L}=W Q^{-1}$ and using the Schur complement, we obtain inequality (20). This ends the proof item i) of this theorem.

With the feedback control (19), the solution of (12) is given by

$$
\mathbf{T}(t)=e^{(\mathbb{A}-\mathbb{B L}) t} \mathbf{T}_{0}+\int_{0}^{t} e^{(\mathbb{A}-\mathbb{B L})(t-s)} f(\mathbf{T}(s)) \mathrm{d} s
$$

The gain matrix, $\mathbb{L}$, can be chosen such that all eigenvalues of matrix $\mathbb{A}-\mathbb{B L}$ have a strictly negative real part.

Then there exist two reals, $M>0$ and $\omega<0$, such that relation (21) holds and $\mathbf{T}(t)$ can be bounded as

$$
\begin{aligned}
\|\mathbf{T}(t)\| & \leqslant M e^{\omega t}\left\|\mathbf{T}_{0}\right\|+M e^{\omega t} \int_{0}^{t} e^{-\omega s} f(\mathbf{T}(s)) \mathrm{d} s . \\
& \leqslant M e^{\omega t} \varepsilon_{0}+M e^{\omega t} \int_{0}^{t} e^{-\omega s} f(\mathbf{T}(s)) \mathrm{d} s .
\end{aligned}
$$

where $\varepsilon_{0}$ is defined in relation (22).

By Lipschitz condition, we have

$$
\|\mathbf{T}(t)\| \leqslant M e^{\omega t} \varepsilon_{0}+M e^{\omega t} \int_{0}^{t} \lambda e^{-\omega s}\|\mathbf{T}(s)\| \mathrm{d} s,
$$

or equivalently as

$$
\|\mathbf{T}(t)\| e^{-\omega t} \leqslant M \varepsilon_{0}+\lambda M \int_{0}^{t} e^{-\omega s}\|\mathbf{T}(s)\| \mathrm{d} s .
$$

Inequality (36) can be written as

$$
\|\mathcal{T}(t)\| \leqslant M \varepsilon_{0}+\lambda M \int_{0}^{t}\|\mathcal{T}(s)\| \mathrm{d} s,
$$

where $\mathcal{T}(t)=\mathbf{T}(t) e^{-\omega t}$.

Hence, using the second Gronwall-Bellman lemma yields the following inequality:

$$
\|\mathcal{T}(t)\| \leqslant M \varepsilon_{0} \exp (\lambda M t) .
$$

Using the fact that $\mathcal{T}(t)=\mathbf{T}(t) e^{-\omega t}$, we then obtain the following inequality:

$$
\|\mathbf{T}(t)\| \leqslant M \varepsilon_{0} \exp (\lambda M+\omega) t .
$$

It is clear that if

$$
\omega<-\lambda M,
$$

then $\lim _{t \rightarrow \infty} \mathbf{T}(t)=0$ which implies the closed-loop system (12) is exponentially stable. This ends the proof item ii) of this theorem.

\section{B. Observer design}

Temperature measurements inside DCMD module are very limited and sometimes inaccessible, however they are extremely needed for the controller to function properly. Therefore, we use stabilizing feedback state observers to provide an estimate for the temperature distribution along the DCMD domain. These observers are derived by using the LMIs and Gronwall-Bellman lemma approaches.

Consider the nonlinear observer in the following form:

$$
\left\{\begin{array}{l}
\dot{\hat{\mathbf{T}}}=\mathbb{A} \widehat{\mathbf{T}}+f(\widehat{\mathbf{T}})+\mathbb{B} U+\mathbb{G}(\zeta-\widehat{\zeta}), \\
\widehat{\zeta}=\mathbb{H} \widehat{\mathbf{T}},
\end{array}\right.
$$

where $\widehat{\mathbf{T}} \in \mathbb{R}^{n}$ is the estimated state vector, $\widehat{\zeta} \in \mathbb{R}^{m}$ is the estimated output vector, $G$ is a observer gain matrix.

Then, the observer error dynamic equation is given by

$$
\dot{e}=(\mathbb{A}-\mathbb{G} \mathbb{H}) e+f(\mathbf{T})-f(\widehat{\mathbf{T}}),
$$

where $e=\mathbf{T}-\widehat{\mathbf{T}}$ is the state estimation error.

The following theorem gives sufficient conditions for the asymptotic and exponential stability of the state observer error (42).

Theorem 2:

i) An asymptotically stable observer of the form (41) exists, if and only if there a symmetric positive definite matrix $S$, a matrix $R$, and a positive scalar $\rho>0$ such that the following linear matrix inequality (LMI) is satisfied

$$
\left[\begin{array}{cc}
\mathbb{A}^{T} S+S \mathbb{A}-R \mathbb{H}-\mathbb{H}^{T} R^{T}+\rho \lambda^{2} I & S \\
S & -\rho I
\end{array}\right]<0 .
$$

The stabilizing observer gain matrix is given by

$$
\mathbb{G}=S^{-1} R \text {. }
$$

ii) However, an exponential stable observer of the form (41) exists, if all eigenvalues of matrix $\mathbb{A}-\mathbb{G} \mathbb{H}$ has a strictly negative real part. Then, two reals, $M_{1}>0$ and $\omega_{1}<-\lambda M_{1}<0$ would be such that

$$
\left\|e^{(\mathbb{A}-\mathbb{G} \mathbb{H}) t}\right\|<M_{1} e^{\omega_{1} t} \quad \forall t \geqslant 0,
$$

and if

$$
0<\left\|e_{0}\right\| \leqslant \varepsilon_{1}
$$

where $\varepsilon_{1}>0$ is a small scalar.

Moreover, the state observer error, $e(t)$, is bounded by

$$
\begin{aligned}
\|e(t)\| & =\|\mathbf{T}(t)-\widehat{\mathbf{T}}(t)\| \\
& \leqslant M_{1} \varepsilon_{1} \exp \left(\lambda M_{1}+\omega_{1}\right) t .
\end{aligned}
$$

Proof: Define $e=\mathbf{T}-\widehat{\mathbf{T}}$ the state estimation error and $V=e^{T} S e$ the Lyapunov candidate function, then $V$ is positive-definite and its derivative along system (42).

$$
\dot{V} \leqslant e^{T}\left((\mathbb{A}-\mathbb{G} \mathbb{H})^{T} S+S(\mathbb{A}-\mathbb{G} \mathbb{H})+\rho^{-1} S S+\rho \lambda^{2} I\right) e .
$$

\section{If}

$$
(\mathbb{A}-\mathbb{G} \mathbb{H})^{T} S+S(\mathbb{A}-\mathbb{G} \mathbb{H})+\rho^{-1} S S+\rho \lambda^{2} I<0,
$$

then $\dot{V}<0$, which implies that the closed-loop system (27) is asymptotically stable.

Taking into account the inequality (48), we define $\mathbb{G}=$ $S^{-1} R$. Using the Schur complement, we obtain inequality (43). Thus, the proof item i) is completed. 
The error dynamic equation (42) can be written as

$e(t)=e^{(\mathbb{A}-\mathbb{G} \mathbb{H}) t} e_{0}+\int_{0}^{t} e^{(\mathbb{A}-\mathbb{G} \mathbb{H})(t-s)}(f(\mathbf{T}(s))-f(\widehat{\mathbf{T}}(s))) \mathrm{d} s$.

Using the Lipschitz condition (18) and applying the second Bellman-Gronwall lemma yields

$$
\|e(t)\| \leqslant M_{1} \varepsilon_{0} \exp \left(\lambda M_{1}+\omega_{1}\right) t,
$$

where $M_{1}$ and $\omega_{1}$ are defined by the relation

$$
\left\|e^{(\mathbb{A}-\mathbb{G} H) t}\right\|<M_{1} e^{\omega_{1} t} \quad \forall t \geqslant 0 .
$$

From (50) it is clear that if

$$
\omega_{1}<-\lambda M_{1}
$$

then $\lim e(t)=0$, which implies the closed-loop observer error $(4 \overrightarrow{4})$ is exponentially stable. This ends the proof item ii) of this theorem.

Remark 1: Since, it is well known that there exists $M$ such that $\left\|e^{A t}\right\| \leqslant M e^{\omega t}, \forall t>0$, where $\omega<0$ is the largest real part of the eigenvalues of matrix $A$, we can maximize $|\omega|$ by solving the following LMI (see [30], p. 66)):

$\max \kappa \quad$ such that $P=P^{T}>0$ and $A^{T} P+P A+2 \kappa P \leqslant 0$ where $\kappa=-\omega$. The previous LMI can be included in the design procedure by replacing $A$ by $\mathbb{A}-\mathbb{B L}$ or $\mathbb{A}-\mathbb{G} \mathbb{H}$.

\section{Observer based output feedback control}

Heat transfer mechanisms, inside the DCMD process, need sufficient temperature difference along the membrane boundary layers. In this regard, observer-based output feedback control is proposed to force the temperature difference to track a desired reference that guarantees stable production rate.

We define the linear combination of the temperature difference between the membrane boundary layers as in (53).

$$
y=\mathbb{C T},
$$

where $y \in \mathbb{R}^{n \times 1}$, and $\mathbb{C} \in \mathbb{R}^{n \times 2 n^{2}}$ is in (54).

$$
\mathbb{C}=\left[\begin{array}{ccccccccc}
0 & \cdots & 1 & 0 & -1 & \cdots & 0 & \cdots & 0 \\
0 & \cdots & 0 & 1 & \cdots & -1 & 0 & \cdots & 0 \\
0 & \cdots & 0 & 0 & \ddots & 0 & \ddots & 0 & 0 \\
0 & \cdots & 0 & \cdots & 0 & 1 & \cdots & -1 & 0
\end{array}\right] .
$$

Using the observer design in theorem 2, we derive a solution to the output feedback control problem for system (12). Our goal here is to design a suitable control law, $U=-\mathbb{L}\left(\mathbb{C} \widehat{\mathbf{T}}+U_{r}\right)$ with $U_{r}$ is the desired temperature difference reference, such that the system (12) is asymptotically stable, and the temperature difference $(y)$ tracks $\left(U_{r}\right)$ such as $\lim _{t \rightarrow \infty} y(t)=U_{r}$.

Fig. 2 depicts the structure of the proposed observerbased output feedback control. The following theorem gives sufficient conditions for the asymptotical stabilization of the system (12).

Theorem 3: The system (12) controlled by the feedback control law

$$
U=-\mathbb{L}\left(\mathbb{C} \widehat{\mathbf{T}}+U_{r}\right),
$$

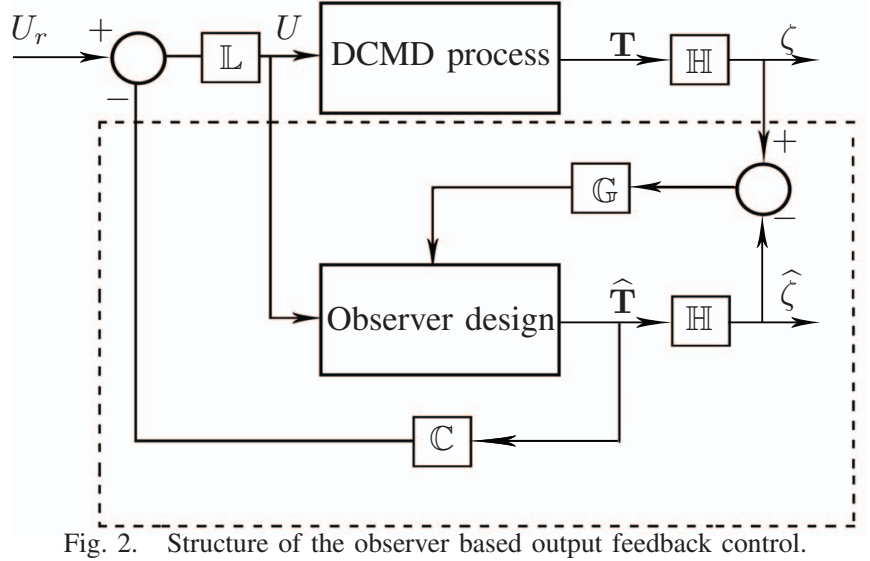

is asymptotically stable, if the LMIs (20) and (43) hold, where $\widehat{\mathbf{T}}$ is the estimate state of $\mathbf{T}$ given by (41), $\mathbb{L}$ is the gain matrix given by $\mathbb{L}=W Q^{-1}$ with $W$ and $Q$ obtained from (20).

Proof: By the definition of the observer error $e=\mathbf{T}-$ $\widehat{\mathbf{T}}$, we obtain the closed-loop of system (12) under the control law (55)

$$
\dot{\mathbf{T}}=(\mathbb{A}-\mathbb{B} \mathbb{L} \mathbb{C}) \mathbf{T}+\mathbb{B L}\left(\mathbb{C} e+U_{r}\right)+f(\mathbf{T}) .
$$

The time derivative of the Lyapunov function candidate given by (28) along the trajectories of system (56) satisfies

$\dot{V} \leqslant \mathbf{T}^{T}\left((\mathbb{A}-\mathbb{B L} \mathbb{C})^{T} P+P(\mathbb{A}-\mathbb{B L} \mathbb{C})+\varepsilon^{-1} P P+\varepsilon \lambda^{2} I\right) \mathbf{T}$

$$
+2 \mathbf{T}^{T} P \mathbb{B L}\left(\mathbb{C} e+U_{r}\right) \text {. }
$$

Let

$$
H=(\mathbb{A}-\mathbb{B L} \mathbb{C})^{T} P+P(\mathbb{A}-\mathbb{B} \mathbb{C} C)+\varepsilon^{-1} P P+\varepsilon \lambda^{2} I<0 .
$$

Using the "W-problem" formulated in Theorem 1 of [32], we obtain the following equation :

$$
\dot{V} \leqslant \lambda_{\max }(H)\|\mathbf{T}\|^{2}+2\|P \mathbb{B} \mathbb{L}\|\|\mathbf{T}\|\left(\|\mathbb{C} e\|+\left\|U_{r}\right\|\right),
$$

or equivalently as

$$
\dot{V} \leqslant m_{1} V+m_{2} \sqrt{V}\|e\|+m_{3} \sqrt{V}\left\|U_{r}\right\|,
$$

where

$$
m_{1}=\frac{\lambda_{\max }(H)}{\lambda_{\min }(P)}, m_{2}=\frac{2\|P \mathbb{B L} \mathbb{C}\|}{\sqrt{\lambda_{\min }(P)}} \text { and } m_{3}=\frac{2\|P \mathbb{B L}\|}{\sqrt{\lambda_{\min }(P)}} .
$$

As a consequence,

$$
\frac{\mathrm{d} \sqrt{V}}{\mathrm{~d} t} \leqslant \frac{m_{1}}{2} \sqrt{V}+\frac{m_{2}}{2}\|e\|+\frac{m_{3}}{2}\left\|U_{r}\right\| .
$$

Note that $m_{1}<0$ and $m_{3}<0$ and from theorem 2, it has been shown that the estimation error converges to zero asymptotically. It follows from (61) that the state $\mathbf{T}$ converges asymptotically to the desired reference value. This ends the proof.

\section{NUMERICAL SIMULATIONS}

In this section, simulations are given to illustrate the performance of the proposed observer-based output feedback control to the DCMD model. The simulations are done with real process parameters values (see [6], [9]) which are provided by the Water Desalination and Reuse Center 
(WDRC) at KAUST. In the plots, each curve represents the temperature difference along the membrane boundary layers.

Fig. 3 shows the tracking of the desired temperature difference reference. In which the controller succeeds to force the temperature difference $(y)$ to track the desired reference (e.g. $U_{r}=8{ }^{\circ} \mathrm{C}$ in the simulations), and thus manages to maintain the production rate of the DCMD process at certain level $\left(\right.$ i.e.8.7872 $\left.\frac{\mathrm{kg}}{\mathrm{m}^{2} \mathrm{~h}}\right)$.

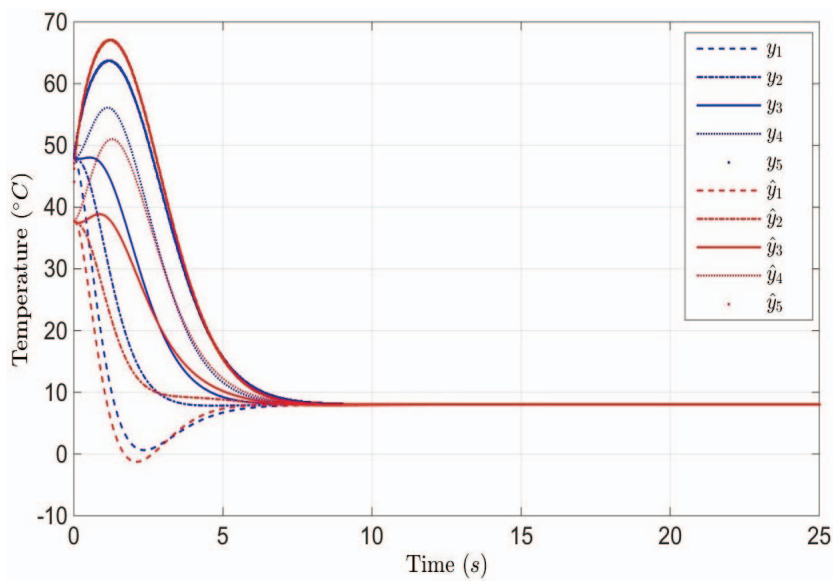

Fig. 3. Reference temperature difference tracking.

\section{CONCLUSION}

In this paper, feedback stabilization for Direct-Contact Membrane Distillation (DCMD) process has been derived using Gronwall-Bellman lemma and LMI formulation. Both approaches present sufficient conditions for the asymptotic and exponential stabilization. Then, an observer design is proposed to provide an estimate for the temperature distribution among the designated process domain, especially at the membrane surfaces. Afterwards, an observer-based output feedback is designed to maintain the estimated temperature difference between the membrane boundary layers fixed at a sufficient reference for stable and feasible operation. The proposed control is simple to implement in practice. Numerical simulations with real process parameters values are presented to show the effectiveness of the proposed approach.

\section{ACKNOWLEDGEMENT}

The research reported herein is supported by the King Abdullah University of Science and Technology (KAUST).

\section{REFERENCES}

[1] M. Khayet, Advanced Membrane Technology and Applications. John Wiley and Sons, Inc., 2008.

[2] L. Camacho, L. Dumee, J. Zhang, J. Li, M. Duke, J. Gomez, and S. Gray, "Advances in membrane distillation for water desalination and purification applications," Water, vol. 5, no. 1, pp. 94-196, 2013.

[3] L. Francis, N. Ghaffour, A. S. Alsaadi, S. P. Nunes, and G. L. Amy, "Performance evaluation of the dcmd desalination process under bench scale and large scale module operating conditions," Journal of Membrane Science, vol. 455, pp. 103-112, 2014.

[4] F. Eleiwi and T. Laleg, "Membrane distillation process modeling: dynamical approach," International Journal of Chemical, Nuclear Metallurgical and Materials Engineering., vol. 8, no. 6, pp. 479-484, 2014
[5] M. Gryta, Water Desalination by Membrane Distillation, ch. Desalination, Trends and Technologies, p. doi: 10.5772/14746. InTech, 2008.

[6] E. Fadi and T. Laleg, "Nonlinear lyapunov-based boundary control of distributed heat transfer mechanisms in membrane distillation plant," in Proc. IEEE American Contr. Conf., (Chicago, USA), 2015.

[7] A. R. Bartman, C. W. McFall, P. D. Christofides, and Y. Cohen, "Model predictive control of feed flow reversal in a reverse osmosis desalination process," in Proc. IEEE American Contr. Conf., (Orlando, USA), 2009.

[8] C. Lawson and R. Hanson, Solving Linear Least Squares Problems. Englewood Cliffs, New Jersey: Prentice Hall, 1974.

[9] E. Fadi and T. Laleg, "Dynamic modeling and optimization in membrane distillation system," in The 19th World Congress of the International Federation of Automatic Control, (South Africa), pp. 33273332, 2014.

[10] M. España and I. Landau, "Reduced order bilinear models for distillations columns," Automatica, vol. 14, pp. 345-355, 1977.

[11] R. Longchamp, "Stable feedback control of bilinear systems," IEEE Trans. Aut. Contr., vol. 25, pp. 302-306, 1980.

[12] R. Mohler, Nonlinear systems : Applications to Bilinear Control, vol. 2. Englewood Cliffs, New Jersey: Prentice Hall, 1991.

[13] J. Jacobson, "Stabilization and optimal control of nonlinear systems homogenous in the input," in Proc. Conf. on Direction in Decentralized Control, (Boston, USA), 1975.

[14] V. Jurdjevic and J. Quinn, "Controllability and stability," J. of Differential Equations, vol. 28, pp. 381-389, 1978

[15] M. Slemrod, "Stabilization of bilinear control systems with applications to nonconservative problems in elasticity," SIAM J. Contr. Opt., vol. 16, pp. 131-141, 1978.

[16] E. Ryan and N. Buckingham, "On asymptotically stabilizing feedback control of bilinear systems," IEEE Trans. Aut. Contr., vol. 28, pp. $863-$ 864, 1983.

[17] A. Benallou, D. Mellichamp, and D. Seborg, "Charaterization of equilibrium sets for bilinear systems with feedback control," Automatica, vol. 19, pp. 183-189, 1983.

[18] J. Gauthier, H. Hammouri, and S. Othman, "A simple observer for nonlinear systems, applications to bioreactors," IEEE Trans. Aut. Contr., vol. 37, pp. 875-880, 1992.

[19] R. Mota, M. Nadri, and H. Hammouri, "Nonlinear implicit on-line observer : application to the estimation in binary distillation columns," in Proc. Triennal IFAC World Congress, (Seoul, Korea), 2008.

[20] B. Pachpatte, "A note on Gronwall-Bellman inequality," Journal of Mathematical Analysis and Applications, vol. 44, pp. 758-762, 1973.

[21] C. Desoer and M. Vidyasagar, Feedback Systems Input-Output Properties. New York: Electrical Sciences. Academic Press, 1975.

[22] M. Vidyasagar, Nonlinear Systems Analysis. Englewood Cliffs, New Jersey: Prentice Hall, 2nd ed., 1993.

[23] A. Zevin and M. Pinsky, "Exponential stability and solution bounds for systems with bounded nonlinearities," IEEE Trans. Aut. Contr. vol. 48, pp. 1799-1804, 2003.

[24] K. Shimizu, "Nonlinear state observers by gradient descent method," in Proc. IEEE Conf. Contr. \& Applications, (Anchorage, USA), 2000.

[25] S. Żak, "On the stabilization and observation of nonlinear/uncertain dynamic systems," IEEE Trans. Aut. Contr., vol. 35, pp. 604-607, 1990.

[26] B. Chen and C. Wong, "Robust linear controller design: time domain approach," IEEE Trans. Aut. Contr., vol. 32, pp. 161-164, 1987.

[27] L. Jetto and V. Orsini, "Relaxed sufficient conditions for the stability of continuous and discrete-time linear time-varying systems," in Proc. IEEE Conf. Decision \& Contr., (New Orleans, USA), 2007.

[28] D. Debeljković and N. Kablar, "Finite-time stability of linear singular systems: Bellman-Gronwall approach," in Proc. IEEE Conf. Decision \& Contr., (San Diego, USA), 1999.

[29] I. N'Doye, M. Zasadzinski, M. Darouach, N. Radhy, and A. Bouaziz, "Exponential stabilization of a class of nonlinear systems : a generalized Bellman-Gronwall lemma approach," Nonlinear Analysis: Theory Methods \& Applications, vol. 74, pp. 7333-7341, 2011.

[30] S. Boyd, L. El Ghaoui, E. Féron, and V. Balakrishnan, Linear Matrix Inequality in Systems and Control Theory. Philadelphia: SIAM, 1994.

[31] M. Pourgholi and V. J. Majd, "A nonlinear adaptive resilient observer design for a class of lipschitz systems using LMI," Circuits, Systems, and Signal Processing, vol. 30, pp. 1401-1415, 2011.

[32] C. Crusius and A. Trofino-Neto, "Sufficient LMI conditions for output feedback control problems," IEEE Trans. Aut. Contr., vol. 44, pp. 1053-1057, 1999. 